\title{
Complicaciones gastrointestinales severas potencialmente relacionadas con el consumo de antiinflamatorios no esteroideos: coste del tratamiento hospitalario para el Sistema Nacional de Salud en nuestro país
}

\author{
E. VARGAS, L. CABRERA, A. MORÓN*, M. GARCÍA, J. SOTO**, J. REJAS*** \\ Servicio de Farmacología Clínica. Hospital Clínico-Madrid. *Servicio de Farmacia. \\ Hospital Parc Tauli. Sabadell. Barcelona. **Unidad de Farmacoeconomía e \\ Investigación de Resultados en Salud. Dpto. de Medicina. Pharmacia, S.A. Madrid. \\ ***Unidad de Investigación de Resultados en Salud. Dpto. Médico. Pfizer, S.A. Madrid
}

\begin{abstract}
SEVERE GASTROINTESTINAL COMPLICATIONS POTENTIALLY RELATED TO THE CONSUMPTION OF NON-STEROIDAL ANTIIN FLAMMATORY DRUGS: HOSPITAL COST DUE TO THEIR TREAT MENT FOR THE NATIONAL HEALTH SYSTEM IN OUR COUNTRY
\end{abstract}

\section{RESUMEN}

Fundamento: Los antiinflamatorios no esteroideos (AINE) producen complicaciones gastro-intestinales (G-I) severas en el 1-4 \% de los casos, las cuales necesitan ser tratadas en el hospital.

El objetivo de este estudio ha sido evaluar el coste hospitalario derivado de tratar estas complicaciones en nuestro Sistema Nacional de Salud (SNS).

Material y métodos: En una primera fase se realizó un estudio transversal para conocer el número de pacientes que ingresaron durante 1998 en dos hospitales terciarios de nuestro país por una complicación G-I severa, revisándose la historia clínica para conocer sí habían consumido algún AINE. En los casos positivos, se anotaron los recursos consumidos durante su hospitalización.

En una segunda fase se estimó el número de hospitalizaciones ocurridas por la misma causa en el conjunto de hospitales de agudos en todo el SNS durante 1998

Resultados: En los dos hospitales estudiados, el 38,1\% de los pacientes ingresados por una complicación G-I severa habían tomado algún AINE en el mismo día o en los días previos. El coste/paciente resultante de su tratamiento ascendió a 389.831 pesetas.

En todo el SNS durante 1998 hubo 54.623 hospitalizaciones por la misma razón. Asumiendo que el $38,1 \%$ de todos los ingresos también hubieran consumido algún AINE, 20.811 pacientes habrían sufrido una complicación G-I severa potencialmente debida a la ingesta de AINE.

Extrapolando el coste/paciente obtenido en los dos hospitales estudiados al global de pacientes ingresados en el SNS, el coste del tratamiento de todas las complicaciones G-I severas relacionadas con el consumo de AINE en nuestro país durante 1998, ascendió a 8.112 millones de pesetas.

Conclusiones: Teniendo en cuenta la alta prevalencia en nuestro medio de la patología osteo-articular, y el alto consumo de AINE para su tratamiento, la llegada de nuevas opciones terapéuticas con un mejor perfil de seguridad G-I supondría un importante ahorro de recursos para el SNS de nuestro país.

PALABRAS CLAVE: Complicaciones gastro-intestinales severas. Ingesta AINE. Coste tratamiento hospitalario.

\section{ABSTRACT}

Background: Non-steroidal antiinflammatory drugs (NSAIDs) produce severe gastrointestinal (G-I) complications in 1-4\% of cases which need to be treated into the hospital.

The aim of this study has been to assess the hospital cost secondary to treat these complications in our National Health Service (NHS).

Material and methods: In the first phase a cross-sectional study was performed in order to know the number of patients who were hospitalized due to a severe G-I complication during 1998 in two tertiary hospital in our country. It was reviewed their clinical charts to know whether they had taken any NSAIDs.

In those positive cases all resources used during the hospitalization were collected.

Results: In both hospitals studied $38.1 \%$ of hospitalized patients for a severe G-I complication had taken any NSAIDs during the same day or previous days. The cost/patient was of 389,831 pesetas.

During 1998 in the whole NHS there were 54,623 hospitalizations owing to the same reason. Assuming that $38.1 \%$ of them had also taken any NSAIDs, 20,811 patients would have suffered a severe G-I complication potentially due to the intake of NSAIDs.

Extrapolating the cost/patient obtained in both hospitals to the global number of patients hospitalized into the NHS, the cost of treting all severe G-I complications related to the consumption of NSAIDs during 1998 was of 8,112 millions pesetas

Conclusions: Bearing in mind the elevated prevalence of osteo-articular pathology in our country and the high consumption of NSAIDs for its treatment, the coming of new therapeutic options with a better safety profile would mean an important resources' saving for our NHS.

KEY WORDS: Severe gastrointestinal complication. Intake of NSAIDs. Hospital cost treatment.

Vargas E, Cabrera L, Morón A, García M, Soto J, Rejas J. Complicaciones gastrointestinales severas potencialmente relacionadas con el consumo de antiinflamatorios no esteroideos: coste del tratamiento hospitalario para el Sistema Nacional de Salud en nuestro país. An Med Interna (Madrid) 2001; 18: 564-568.

Trabajo aceptado: 6 de junio de 2001

Correspondencia: J. Soto Álvarez. Pharmacia, S.A. Avda de Burgos 17-1ª Planta. 28036 Madrid 
Los antiinflamatorios no esteroideos (AINEs) son ampliamente usados en la práctica médica habitual para el tratamiento sintomático de procesos músculo-esqueléticos y osteo-articulares.

En nuestro país durante 1998 se recetaron más de 30 millones de envases de AINE, a lo que habría que añadir el consumo como automedicación al margen de la prescripción facultativa (1).

No obstante, su empleo no está libre exento de que puedan aparecer reacciones adversas (RAs), siendo las más frecuentes y graves las que se producen en el tracto gastro-intestinal (G-I) (2). Así, se ha comprobado que entre los pacientes tratados con AINE, el 10-20\% sufrirán dispepsia, el 5-30\% en tratamiento crónico desarrollarán ulcera péptica, y entre el 1-4\% presentarán complicaciones G-I severas (hemorragias, perforaciones u obstrucciones), siendo necesaria la hospitalización para su tratamiento $(3,4)$. Van a existir unos factores que van a favorecer la aparición de RAs cuando se ingiera un AINE, como son la edad (por encima de 60 años), historia previa de ulcera péptica o sangrado G-I, ingesta dosis altas de AINE y uso concomitante de anticoagulantes y corticoides $(5,6)$.

De hecho, en varios trabajos se ha visto que la toma de AINE y el posterior desarrollo de Ras y complicaciones G-I graves, han sido una de las mayores causas de ingreso hospitalario (7-9), originando un importante consumo de recursos (derivado de su diagnóstico y tratamiento) para los Sistemas Sanitarios (10).

El objetivo de nuestro estudio, ha sido evaluar la repercusión económica para la asistencia hospitalaria del Sistema Nacional de Salud (SNS) de nuestro país, derivado del ingreso y manejo de pacientes con complicaciones G-I severas, potencialmente relacionadas con la ingesta de AINE.

\section{MATERIAL Y MÉTODOS}

El estudio tuvo dos fases bien diferentes. Inicialmente, se diseño un estudio descriptivo transversal con el objeto de detectar los ingresos hospitalarios ocurridos durante 1998 (en dos hospitales terciarios de nuestro país: Hospital Clínico de Madrid y Parc Tauli de Sabadell) debidos a una complicación G-I severa. Una vez detectados, se revisaron retrospectivamente las historias clínicas de los pacientes en los que la complicación potencialmente hubiera estado relacionada con el consumo de cualquier AINE, incluyendo las especialidades farmacéuticas publicitarias.

Para su detección se revisó el diagnóstico principal del Conjunto Mínimo Básico de Datos (CMBD) de todas las altas de los dos hospitales durante 1998, considerándose como posible ingreso debido a una complicación G-I, a los pacientes que hubiesen tenido alguno de los siguientes diagnósticos, clasificados según la Clasificación Internacional de Enfermedades de la OMS, $9^{\mathrm{a}}$ revisión modificación clínica $3^{\mathrm{a}}$ edición española (CIE9-MC): enfermedad del esófago (530,1, 530,2, 530,8), úlcera de estómago $(530,0,531,9)$, úlcera de duodeno (532,0-532.9), úlcera péptica $(533,0-533,9)$, úlcera gastro-yeyunal $(534,0-534,9)$, gastritis, gastroduodenitis, gastro-yeyunitis $(535,0,535,4$, $535,5)$, hemorragia duodeno $(537,89)$, hemorragia estómago (578), hematémesis y melenas $(578,0,578,1)$.

Una vez identificados todos los pacientes ingresados por alguna de estas patologías, se solicitaron sus historias clínicas, las cuales fueron revisadas manualmente una a una para cono- cer si en el momento de su ingreso (o durante los 15 días previos), los pacientes habían consumido algún tipo de AINE, tanto de prescripción como de autoadministración. La revisión de las historias fue realizada por miembros del Servicio de Archivo y Documentación Clínica de ambos hospitales (3 en un hospital y 2 en el otro), anotando toda la información en un cuaderno de recogida de datos diseñado para tal fin, donde se recopilaron datos administrativos y clínicos de interés, así como todos los datos de los recursos consumidos durante la estancia hospitalaria de los pacientes que estaban tomando algún AINE en el momento de aparecer el evento. Por limitación de tiempo y escasez de recursos humanos, no se realizó ningún proceso para validar y evaluar la calidad de los datos recogidos.

En los pacientes en los que se descubrió una ingesta de cualquier AINE en el momento del ingreso (o durante los 15 días previos), se revisaron fehacientemente las historias clínicas, recogiéndose los recursos que fueron necesarios para el diagnóstico y tratamiento de las complicaciones G-I severas durante su estancia hospitalaria. Se incluyó: medicación administrada, procedimientos quirúrgicos, consultas intrahospitalarias a especialistas, pruebas diagnósticas y analíticas realizadas, administración de hemoderivados y días de estancia en UCI y planta de hospitalización.

El coste de la medicación empleada se obtuvo del Catálogo de Especialidades Farmacéuticas (Editado por el Consejo General de Colegios Oficiales de Farmacéuticos ) del año 2000 (habiéndose escogido el precio de venta del laboratorio-PVL), mientras que el coste del resto de recursos consumidos se recabó en cada hospital participante (derivado de su contabilidad analítica) habiéndose expresado todo en pesetas de 2000.

En una segunda fase, se estudio la incidencia de complicaciones G-I severas que motivaron ingreso hospitalario en todo el SNS durante 1998. Para su evaluación, se empleo la base de datos de Iasist (agencia externa de provisión de información para gestión clínica, con la siguiente dirección en Internet: http://www.iasist.com), la cual en aquel momento estaba compuesta de los datos del CMBD correspondiente a los episodios de alta hospitalaria de 79 hospitales de agudos ocurridos durante 1998.

Dado el gran número de hospitales incluidos en esta base de datos, su distribución por tipo de hospital y el volumen de datos incluidos (alrededor del 40\% de todos los episodios de hospitalización de los hospitales de agudos del SNS estaban recogidos), se ha asumido que estos datos son una buena representación del total de episodios de los hospitales generales de agudos de nuestro país. Con los resultados hallados en la revisión de esta base de datos, y extrapolando los hallazgos al conjunto del SNS, se estimó que el total de episodios de hospitalización en centros generales públicos de agudos en el SNS fue de 3.454.722 durante 1998.

Se revisó el diagnóstico principal del CMBD de todos estos ingresos, recogiéndose como ingreso por complicación G-I severa los mismos diagnósticos (clasificados según la CIE-9-MC) que fueron evaluados en la $1^{\text {a }}$ fase del estudio en los 2 hospitales estudiados.

Para la caracterización de los episodios de hospitalización (tanto en los dos hospitales estudiados en la primera fase como en el conjunto global del SNS), se aplicó como sistema de clasificación de pacientes los GRD o grupos relacionados con el diagnóstico. Los GRD son un sistema de clasificación de episodios de hospitalización que tienen como principal 
característica la homogeneidad clínica y el consumo de recursos de los episodios de cada clase. Cada episodio de hospitalización es clasificado en un GRD de manera unívoca de acuerdo con el diagnóstico principal, entidad patológica que motiva el ingreso en el hospital, la presencia o ausencia de intervenciones quirúrgicas y de otras variables (edad, complicaciones o co-morbilidades presentes y estado de alta). Este sistema está ampliamente extendido y validado en la mayoría de países occidentales y en nuestro entorno $(11,12)$.

\section{RESULTADOS}

En la tabla I se detalla el número de ingresos durante 1998 en ambos hospitales y en el conjunto global del SNS, el porcentaje con complicaciones G-I severas y la proporción de estos pacientes que habían consumido algún AINE previo al ingreso.

\section{TABLA I}

\begin{tabular}{|c|c|c|}
\hline \multicolumn{3}{|c|}{ TABLA I } \\
\hline \multicolumn{3}{|c|}{$\begin{array}{c}\text { PORCENTAJE DE PACIENTES INGRESADOS } \\
\text { POR CO M PLICACIO NES G-I SEVERAS DURANTE } 1998 \\
\text { EN LOS DOS HOSPITALES EVALUADOS Y EN EL CO NJUNTO } \\
\text { DEL SISTEM A NACIO NAL DE SALUD (SNS), JUNTO } \\
\text { A LA PRO PO RCIÓ N DE TOM ADO RES } \\
\text { DE AINE EN LA FASE PREVIA }\end{array}$} \\
\hline & Hospitales estudiados & Conjunto SNS \\
\hline Total ingresos & 65.065 & 3.457 .151 \\
\hline $\begin{array}{l}\text { Porcentaje complicaciones } \\
\text { G-I severas }\end{array}$ & $0,89 \%(578)$ & $1,58 \%(54.623)$ \\
\hline Proporción tomadores AINE & $=38,1 \%(220 / 578)$ & - \\
\hline
\end{tabular}

G-I: gastro-intestinales

AINE: antiinflamatorio no esteroideo

En la tabla II se exponen los GRD identificados con mayor frecuencia, tanto en los pacientes ingresados por complicaciones G-I severas (y antecedentes de ingesta de AINE) en los 2 hospitales estudiados, como en el global del SNS analizado.

En la tabla III se resume el consumo de recursos derivado del manejo de las complicaciones G-I severas (en aquellos pacientes que previamente habían consumido algún tipo de AINE) en los dos hospitales evaluados, así como la extrapolación al total del SNS durante 1998 (a través de los recursos consumidos por cada GRD), asumiendo que la proporción de pacientes con antecedentes de ingesta de AINE fuese similar al porcentaje hallado en el estudio efectuado en los 2 hospitales.

En la tabla IV, se refleja el análisis de sensibilidad efectuado, variando el valor de aquellas variables que a priori presentan una mayor incertidumbre o tienen un mayor peso en el resultado final del estudio.

\section{DISCUSIÓN}

En la actualidad, es ampliamente reconocido que la ingesta de AINE es uno de los factores que desencadenan compli-
TABLA ॥

GRUPOS RELACIO NADOS CON EL DIAGNÓ STICO (GRD) ESTABLECIDOS EN LOS PACIENTES INGRESADOS

POR COM PLICACIO NES G-I SEVERAS, TANTO EN LA SERIE DE LOS DOS HOSPITALES ESTUDIADOS COM O EN EL CONJUNTO GLOBAL DEL SISTEM A NACIO NAL DE SALUD (SNS)

\begin{tabular}{lcc}
\hline GRD & Hospitales estudiados & Todos el SNS \\
\hline Hemorragia G-I sin complicación & $54,7 \%$ & $48,6 \%$ \\
Hemorragia G-I con complicaciones & $26,7 \%$ & $31,5 \%$ \\
$\begin{array}{l}\text { Procedimientos sobre estómago, } \\
\text { esófago y duodeno con edad >17 }\end{array}$ & & \\
$\begin{array}{l}\text { con complicaciones } \\
\text { Procedimientos sobre estómago, }\end{array}$ & $2,7 \%$ &, $4 \%$ \\
$\begin{array}{l}\text { esófago y duodeno con edad >17 } \\
\text { sin complicaciones }\end{array}$ & $3,3 \%$ & \\
$\begin{array}{l}\text { Esofagitis, gastroenteritis \& trastornos } \\
\text { digestivos misceláneos con edad >17 }\end{array}$ & & \\
con complicaciones & $1,7 \%$ & $2,7 \%$ \\
$\begin{array}{l}\text { Esofagitis, gastroenteritis \& trastornos } \\
\text { digestivos misceláneos con edad >17 } \\
\text { sin complicaciones }\end{array}$ & & \\
Úlcera péptica no complicada sin & $2,1 \%$ &, $1 \%$ \\
complicaciones & & \\
Úlcera péptica complicada & $0,7 \%$ & $2,5 \%$ \\
Úlcera péptica no complicada con & $1,3 \%$ & $1,8 \%$ \\
complicaciones & & $1,7 \%$ \\
\hline
\end{tabular}

TABLA III

CONSUM O DE RECURSOS (PESETAS) ASO CIADO

AL TRATAM IENTO DE LAS CO M PLICACIO NES G-I SEVERAS

EN LOS DOS HOSPITALES REVISADOS, EN LOS PACIENTES

QUE PREVIAM ENTE HABÍAN CONSUMIDO ALGÚN AINE, Y SU GENERALIZACIÓ N AL GLOBAL DEL SISTEM A NACIO NAL DE SALUD (SNS)

\begin{tabular}{|c|c|c|}
\hline & Hospitales evaluados & Global del SNS \\
\hline $\begin{array}{l}\text { Ingresos debidos a } \\
\text { complicaciones G-I severas }\end{array}$ & 578 & 54.623 \\
\hline $\begin{array}{l}\text { Antecedentes ingesta AINE } \\
\text { los días previos }\end{array}$ & 220 & 20.811 \\
\hline $\begin{array}{l}\text { Coste/ paciente } \\
\text { recursos consumidos }\end{array}$ & $\begin{array}{c}389.831 \\
85.762 .857\end{array}$ & $\begin{array}{c}389.831 \\
8.112 .772 .941\end{array}$ \\
\hline
\end{tabular}

caciones G-I severas, las cuales deben ser tratadas siempre en el ámbito hospitalario, aunque parece que influye poco en el desencadenamiento de la anemia ferropénica en pacientes de edad avanzada (13). Así, se ha descrito que el $65 \%$ de los pacientes con complicaciones G-I se asocian con el consumo de aspirina u otro tipo de AINE (14), así como el 32\% de las perforaciones duodenales y el $44 \%$ de las perforaciones gástricas (15). De hecho, se ha estimado que de todos los pacientes que requieren cirugía por complicaciones G-I graves, el $60 \%$ estaban tomando algún tipo de AINE (16). 


\section{TABLA IV}

\begin{tabular}{c} 
TABLA IV \\
ANÁLISIS DE SENSIBILIDAD EFECTUADO \\
CON LOS RESULTADO S GLO BALES DEL SISTEM A \\
NACIONAL DE SALUD (SNS), AL VARIAR AQ UELLOS \\
PARÁM ETROS M ÁS INFLUYENTES EN EL RESULTADO \\
\hline Variables Coste global SNS \\
(pesetas)
\end{tabular}

Mismo porcentaje de complicaciones

G-I severas que las halladas en los 2 hospitales estudiados $(0,89 \%)$

4.569 .931 .635

Porcentaje de pacientes con antecedentes ingesta

AINE un $10 \%$ menor que lo encontrado en los dos hospitales evaluados (34,3\%)

7.303 .752 .378

Porcentaje de pacientes con antecedentes de ingesta AINE un $10 \%$ mayor que la hallada en los dos hospitales estudiados $(41,9 \%)$

Coste/paciente un $10 \%$ más bajo que el hallado en los dos hospitales

7.301 .497 .728

Coste/ paciente un $10 \%$ más lato que el encontrado en los dos hospitales estudiados

En la primera fase de este estudio hemos encontrado que el $0,89 \%$ de los ingresos anuales de los dos hospitales terciarios evaluados son debidos a complicaciones G-I severas, aunque con una diferencia notable de un hospital al otro $(1,14$ vs $0,64 \%$ ). Aunque no esta clara la causa de esta diferencia, una explicación plausible podría ser las distintas características de la población cubierta por ambos hospitales, siendo de mayor edad en el hospital donde se ha encontrado la proporción más elevada. Otra posible causa podría ser la diferente tasa de consumo de AINE/habitante en las dos áreas sanitarias, así como la distinta prevalencia de infección por $H$. pylori en ambas zonas geográficas. Otra posible razón de la discrepancia, podría ser la metodología seguida en ambos hospitales a la hora de codificar los diagnósticos principales en el CMBD de ambos hospitales, y la calidad y claridad de los diagnósticos especificados por los facultativos en las historias clínicas de los pacientes.

Además, hemos hallado que el 38,1\% de los pacientes con ingreso debido a complicaciones G-I severas pueden ser atribuibles a la ingesta de AINE, siendo resultados similares a los descritos en la literatura, donde se puede encontrar que los AINE podrían estar en relación con la hospitalización de pacientes por complicaciones G-I graves en diferentes proporciones: $30 \%$ (17), $49 \%$ (18), $28 \%$ (19) y $44 \%$ (20).

Dado el carácter observacional de nuestro estudio, es imposible asegurar que la ingesta de AINE fue la causa de la complicación G-I en todos los casos (ya que existen otros factores bien conocidos que pueden desencadenar una complica- ción de una úlcera péptica), aunque probablemente en todos ellos el uso del AINE haya sido un factor que ha brá contribuido al desencadenamiento de la complicación. Por otra parte, y dado que el estudio es retrospectivo y que toda la información se ha extraído de las historias clínicas de los pacientes (cuya calidad no siempre es la deseada), es posible que haya habido pacientes en los que no se detectó consumo de AINE en la revisión de la historia (porque en su momento no se recogió esta información) pero que realmente sí los tomaban, básicamente como especialidades farmacéuticas publicitarias (OTC) y a demanda, no de una forma regular, por lo que la implicación de los AINE podría haber sido más elevada de lo encontrado en nuestro estudio.

Para la generalización de los datos, encontrados en los dos hospitales estudiados, al conjunto global del SNS, hemos tenido que asumir que la proporción de pacientes que estarían tomando AINE en el momento de la aparición de la complicación G-I severa, fuera igual al porcentaje hallado en los 2 hospitales estudiados $(38,1 \%)$, lo cual siempre es una posible fuente de sesgos. Sin embargo, este dato se ha incluido en el ulterior análisis de sensibilidad efectuado, lo que nos va a permitir conocer como repercutirá en el resultado final la variación de este porcentaje.

El coste derivado del manejo hospitalario de complicaciones G-I severas en pacientes en tratamiento con AINE, ha sido estudiado en múltiples trabajos y entornos sanitarios, y en todos ellos el consumo de recursos derivado de su tratamiento ha supuesto una cuantía monetaria nada despreciable (21-24). El coste hallado en el estudio de los 2 hospitales evaluados, de 389.831 pesetas/paciente y 85 millones de pesetas durante 1998, sigue la misma línea que los hallazgos de los estudios antes citados hechos en otros países, y es similar al resultado obtenido en un reciente estudio efectuado en nuestro medio, donde el coste del manejo de cada complicación G-I grave supuso un desembolso de 312.654 pesetas/paciente (25). Cuando se ha generalizado el gasto generado en estos 2 hospitales al total del SNS, se ha visto que el montante total de tratar las complicaciones G-I severas (alrededor de 8.000 millones de pesetas anual) supone una cuantía nada despreciable para las arcas del erario público en nuestro país, siendo también muy similar a los valores encontrados en el estudio antes citado, donde se estimó un coste global para el SNS entre 6.094 y 9.497 millones de pesetas anuales.

Teniendo en cuenta la alta prevalencia de enfermedades osteo-articulares en nuestro país, y que el tratamiento sintomático básico de estas dolencias se realiza con AINE, el consumo de recursos derivados de tratar en el hospital las complicaciones G-I severas, va a suponer una cuantía importante para el SNS. Por este motivo, es necesario la búsqueda de nuevas armas terapéuticas que presenten un mejor perfil de seguridad G-I que los AINE convencionales, lo que contribuiría a disminuir el gasto sanitario derivado del manejo de las reacciones adversas ocasionadas por los AINE clásicos a nivel del tracto gastro-intestinal.

\section{Bibliografía}

1. Prieto C, Vargas E. Problemas de uso de los antiinflamatorios no esteroideos (AINE) en pacientes con patología crónica asociada. Inf Ter Sist Nac Salud 2000; 24: 85-91.

2. Cryer B, Kimmay MB. Gastrointestinal side effects of nonsteroidal antiinflammatory drugs. Am J Med 1998; 105: 20S-30S.

3. Wolfe MM, Lichtenstein DR, Singh G. Gastrointestinal toxicity of nonsteroidal antiinflammatory drugs. N Engl J Med 1999; 340: 18881899.

4. Cryer B. NSAID gastrointestinal toxicity. Curr Opin Gastroenterol 2000; 16: 495-502. 
5. Rodríguez C. Factores de riesgo de la gastropatia por AINE. Rev Esp Reumatol 2000; 27: 9-14.

6. Hernández-Díaz S, García Rodríguez LA. Association between nonsteroidal anti-inflammatory drugs and upper gastrointestinal tract bleeding/perforation. Arch Intern Med 2000; 160: 2093-2099.

7. Einarson TR. Drug-related hospital admissions. Ann Pharmacother 1993; 27: 832-840.

8. Major S, Badr S, Bahlawan L, Hassan G, Khogaoghlanian T, Khalil R, et al. Drug-related hospitalization at a tertiary teaching center in Lebanon: incidence, associations and relation to self-medicating behavior. Clin Pharmacol Ther 1998; 64: 450-461.

9. Muñoz MJ, Ayani I, Rodríguez-Sasiain JM, Gutiérrez G, Aguirre C. Monitorización en un servicio de urgencias de reacciones adversas causadas por medicamentos en niños y adultos. Med Clin (Barc) 1998; 111: 92-98.

10. Smalley WE, Griffin MR, Fought RL, Ray WA. Excess costs for gastrointestinal disease among nonsteroidal anti-inflammatory drug users. J Gen Intern Med 1996; 11: 461-469.

11. Casas M. Los Grupos Relacionados con el Diagnóstico. Experiencias y perspectivas de utilización. Masson Editores S.A. Barcelona 1991.

12. Casas M, Wiley MM. Diagnosis related Group in Europe. Uses and perspectives. Springer Verlang, Berlin, 1993.

13. Martínez Rey C, González Quintela A, Domínguez Santalla MJ, Fernández Castroagudín J, Zúñiga Lorenzo V. Patología digestiva alta en pacientes de edad avanzada con enemia ferropénica: comparación entre usuarios y no usuarios de anti-inflamatorios no esteroideos. An Med Interna (Madrid) 2001; 18: 357-360.

14. Wilcox CM, Shelek KA., Cotsonis G. Striking prevalence of over-the counter nonsteroidal anti-inflammatory drug use in patients with upper gastrointestinal hemorrhage. Arch Intern Med 1994; 154: 42-46.
15. Collier DEStJ, Pain JA. Non-steroidal anti-inflammatpory drugs and peptic ulcer perforation. Gut 1985; 26: 359-363.

16. Armstrong CP, Blower AL. Non-steroidal anti-inflammatory drugs and life-threatening complications of peptic ulceration. Gut 1987; 28: 527-532.

17. Blower AL, Brooks A, Fenn GC, Hills A, Pearce MY, Morant S, et al. Emergency admissions for upper gastrointestinal disease and their relation to NSAID use. Aliment Pharmacol Ther 1997; 11: 283-291.

18. Bakowsky VS, Hauly JG. Complications of nonsteroidal antiinflammatory drug gastropathy and use of gastric cytoprotection: experience at a Tertiary Care Health Center. J Rheumatol 1999; 26: 1557-1563.

19. Somerville K, Faulkner G, Langman M. Non-steroidal antiinflammatory drugs and bleeding peptic ulcer. Lancet 1986; 1: 462-464.

20. Lanas A, Serrano P, Bajador E, Estera F, Benito R, Sáinz R. Evidence of aspirin use in both upper and lower gastrointestinal perforation. Gastroenterology 1997; 112: 683-689.

21. Bloom BS. Direct medical costs of disease and gastrointestinal side effects during treatment for arthritis. Am J Med 1988; 84 (Suppl 2A): 20.24.

22. De Pouvourville G. Evaluating the real cost of NSAID therapy: "shadow costs "relating to the treatment of gastrointestinal side effects. Br J Med Econ 1992; 5: 45-50.

23. Kong SX, Hatoum HT, Zhao SZ, Agrawal NM, Geis SG. Prevalence and cost of hospitalization for gastrointestinal complications related to peptic ulcers with bleeding or perforation: comparison of two National Databases. Am J Man Care 1998; 4: 399-409.

24. Moore RA, Phillips CJ. Cost of NSAID adverse effect to the UK National Health Service. J Med Econ 1999; 2: 45-55.

25. Lanas A. Impacto económico de los efectos secundarios gastro-intestinales asociados a antiinflamatorios no esteroideos en el Servicio Nacional de Salud. Med Clin (Barc) 2000; 114 (Supl 3): 46-53. 